\title{
Protección legal a los metadatos y la gestión digital de los derechos de autor
}

\author{
LEGAL PROTECTION TO METADATA AND DIGITAL COPYRIGHT MANAGEMENT
}

JHONNY ANTONIO PABÓN CADAVID ${ }^{1}$

\section{RESUMEN}

Los tratados de la OMPI de 1996 establecen que la integridad de los metadatos de derechos de autor como un nuevo objeto de protección de la propiedad intelectual bajo la categoría de información sobre la gestión de derechos (ISGD). Este artículo analiza desde el ámbito jurídico y de las ciencias de la información el origen de esta clase de tutela jurídica, sus objetivos y fundamentos, así mismo se discute de qué manera la definición de la ISGD adoptada por la OMPI es vaga e imprecisa. El artículo resalta la importancia de los metadatos legales para la circulación eficiente de obras en las redes digitales, incluyendo las obras en el dominio público, el licenciamiento y la gestión de derechos y regalías. Además, se analiza el desarrollo de infraestructuras de información de derechos como parte de las nuevas políticas públicas en materia de propiedad intelectual y como piedra angular para resolver el problema de las obras huérfanas.

\section{ABSTRACT}

The integrity of copyright metadata (information rights management) is a new subject matter for intellectual property. The WIPO treaties of 1996 established that the integrity of the metadata that provide copyright information must be protected. This article discusses from an interdisciplinary perspective the origin of this kind of legal protection, its objectives and foundations. It analyses how WIPO adopted a vague and imprecise definition of the ISGD. The article highlights the importance of legal metadata for the efficient dissemination of works in digital networks, including works in the public domain, licensing and management of rights and royalties. The development of rights information infrastructures is analyzed as part of new public policies on intellectual property and as a cornerstone to solve the orphan works problem.

PALABRAS CLAVE

Derechos de autor, metadatos, licencias, propiedad intelectual, infraestructuras digitales.

\section{KEY WORDS}

Copyright, metadata, licencing, intellectual property, digital infrastructures.

\section{Introducción}

Los metadatos son el ADN de los contenidos culturales en la era digital. Esta es una de las conclusiones del reciente Informe Lescure, encargado por el Ministerio de Cultura de Francia para crear políticas culturales en el ambiente digital ${ }^{2}$. La creciente importancia de los metadatos los ha convertido en un foco de análisis legal y objeto de regulación en diversos contextos, como la

\footnotetext{
${ }^{1}$ Victoria University of Wellington, Wellington, Nueva Zelanda. Email: pabonjhon@myvuw.ac.nz

2 LESCURE (2013), p. 461.
} 
seguridad nacional ${ }^{3}$, la privacidad ${ }^{4}$ y la propiedad intelectual ${ }^{5}$. Además, los metadatos tienen una creciente función en el análisis forense de evidencia digital ${ }^{6}$ y por otro lado son activos informacionales de valor económico regulados y gestionados por contratos ${ }^{7}$. Los metadatos legales son indispensables y esenciales en las actuales soluciones tecnológicas para el uso de contratos inteligentes (smart contracts) y en el uso de tecnologías como blockchain para la gestión de derechos de autor ${ }^{8}$.

Existen varias definiciones del concepto metadato. La definición más común y genérica indica que los metadatos son "datos sobre datos" ${ }^{9}$. Oxford English Dictionary define los metadatos como "un conjunto de datos que describe y proporciona información sobre otros datos" ${ }^{10}$. La literatura académica, desde las ciencias de la información, proporciona las principales definiciones que diversas disciplinas usan del término metadatos. La Organización Nacional de Estándares de Información de los Estados Unidos (NISO) define los metadatos como la "información estructurada que describe, explica, localiza o facilita la recuperación, el uso o la gestión de un recurso informacional" ${ }^{11}$. La característica principal de esta definición es que los metadatos son información sobre recursos de información. La definición de NISO difiere de la de Oxford English Dictionary, que establece que los metadatos son datos sobre datos. Sin embargo, datos, información y conocimiento son tres conceptos diferentes ${ }^{12}$. Los datos potencialmente se pueden convertir en información y la información en conocimiento ${ }^{13}$. La definición de NISO es operacional en el contexto de este artículo ya que los recursos de información digital comprenden creaciones intelectuales protegidas por el derecho de autor, como videos, música y sitios web.

Actualmente, la única protección legal a la integridad de los metadatos es a través de la legislación de derechos de autor, específicamente, la protección que se otorga a la información sobre la gestión de derechos (ISGD). La información sobre la gestión de derechos son metadatos acerca de los derechos de autor y de las licencias de obras protegidas. Esta es una nueva categoría legal cuya protección jurídica garantiza la integridad de los metadatos. Este es un derecho adicional a los titulares de los derechos de autor.

La literatura académica dedicada a analizar la información sobre la gestión de derechos es escaza y en español es inexistente. El presente artículo busca llenar este vacío y responderá a los siguientes interrogantes: ¿qué es la información sobre la gestión de derechos (ISGD)? ¿Cuál es la historia legislativa a nivel internacional de la protección a la ISGD? ¿Cuáles son los objetivos de dicha protección y la función de la ISGD en la gestión digital de contenidos culturales? ¿Cuál es la importancia del desarrollo de infraestructuras tecnológicas para el uso de los metadatos legales? Es así como este artículo procede a resolver estos interrogantes desde una perspectiva interdisciplinaria, desde lo jurídico y las ciencias de la información, de la siguiente manera: primero

\footnotetext{
${ }^{3}$ Ver por ejemplo la legislación reciente de Australia: Telecommunications (Interception and Access) Amendment (Data Retention) Act 2015 (Australia). La regulación de los metadatos con fines de vigilancia es altamente polémica. Ver SCOTT (2015); TAYLOR Y HURST (2015); FARRELL (2014).

${ }^{4}$ BENNET et al. (2014).

${ }^{5}$ Para una perspectiva general ver STROWEL (2000). Los metadatos en algunos casos también pueden ser protegidos por los derechos de bases de datos en los países que existe esta clase de legislación, ver: European Union Directive 96/9/EC 1996 on the Legal Protection of Databases [1996] OJ L 077; Ley Federal del Derecho de Autor de 24 de diciembre de 1996, art 108 (México).

${ }^{6}$ WESCOTT II (2007); ROBINTON (2010), p. 326.

7 JUDGE Y SCASSA (2010).

${ }^{8}$ ANDERS et al. (2018).

${ }^{9}$ NATIONAL LIBRARY OF AUSTRALIA (2003), p. 158.

${ }^{10}$ OXFORD ENGLISH DICTIONARY (2018).

${ }^{11}$ NISO (2004), p. 1.

${ }^{12}$ ZINS (2007).

${ }^{13}$ HJØRLAND (2000), p. 32.
} 
se explorará la definición de información sobre la gestión de derechos, luego se pasará a discutir el punto de partida legislativo de la ISGD en el derecho público internacional y se examinará la justificación normativa para este nuevo conjunto de derechos y sus objetivos. Finalmente, se explorarán las infraestructuras digitales que organizaciones privadas e instituciones públicas están desarrollando para la gestión de metadatos de derechos de autor y su relación con las obras huérfanas.

\section{Información sobre la gestión de derechos}

\subsection{Definición}

La información sobre la gestión de derechos (ISGD) son metadatos adjuntos a obras protegidas por los derechos de autor o metadatos que aparecen en relación con la obra y proporcionan información de propiedad intelectual, como el título de la obra, el autor, el titular de los derechos de autor o las condiciones de uso. En otras palabras, la información sobre la gestión de derechos son metadatos legales relacionados con los derechos morales, los derechos patrimoniales y los términos de las licencias de las obras protegidas por el derecho de autor.

El derecho internacional público define a la ISGD en el Tratado de la OMPI sobre Derecho de Autor (TODA) de la siguiente manera ${ }^{14}$ :

[...] información que identifica a la obra, al autor de la obra, al titular de cualquier derecho sobre la obra, o información sobre los términos y condiciones de utilización de la obras, y todo número o código que represente tal información, cuando cualquiera de estos elementos de información estén adjuntos a un ejemplar de una obra o figuren en relación con la comunicación al público de una obra.

Igualmente, en el Tratado de la OMPI sobre interpretación o ejecución y fonogramas (TOIEF) se define a la ISGD como ${ }^{15}$ :

[...] información que identifica al artista intérprete o ejecutante, a la interpretación o ejecución del mismo, al productor del fonograma, al fonograma y al titular de cualquier derecho sobre interpretación o ejecución o el fonograma, o información sobre las cláusulas y condiciones de la utilización de la interpretación o ejecución o del fonograma, y todo número o código que represente tal información, cuando cualquiera de estos elementos de información esté adjunto a un ejemplar de una interpretación o ejecución fijada o a un fonograma o figuren en relación con la comunicación o puesta a disposición del público de una interpretación o ejecución fijada o de un fonograma.

En el Tratado de la OMPI sobre interpretaciones y ejecuciones audiovisuales de Beijing se define a la ISGD de la siguiente manera ${ }^{16}$ :

[...] información que identifica al artista intérprete o ejecutante, a la interpretación o ejecución del mismo, y al titular de cualquier derecho sobre la interpretación o ejecución, o la información sobre las cláusulas y condiciones de la utilización de la interpretación o ejecución, y todo número o código que represente tal información, cuando cualquiera de estos elementos de información esté adjunto a una interpretación o ejecución fijada en una fijación audiovisual.

\footnotetext{
${ }^{14}$ TODA, art. 12.

${ }^{15}$ TOEIF, art. 19.

${ }^{16}$ OMPI Tratado de Beijing sobre Interpretaciones y Ejecuciones Audiovisuales (2012), art. 16.
} 
Estas definiciones comparten una estructura similar: primero, se refieren a metadatos que proporcionan información de propiedad intelectual; segundo, las definiciones incluyen identificadores (números o códigos) y, tercero, el alcance de la definición es tecnológicamente neutral, se aplica a medios digitales y tradicionales.

La protección a la ISGD se ha estudiado y asociado principalmente con las medidas tecnológicas de protección (MTP $)^{17}$. Por ejemplo, Stephen Fraser incluye la ISGD como parte de los logros de los maximalistas en la protección de derechos de autor debido al vínculo de la ISGD con las MTP y con los sistemas de gestión de derechos digitales (digital rights management -DRM-) ${ }^{18}$. Sin embargo, es necesario aclarar que los sistemas de gestión de derechos (DRM), la ISGD y las MTP son tres objetos diferentes. De manera que todos los DRM incluyen ISGD, pero no necesariamente MTP. Mihály Ficsor acertadamente advierte que cuando se critica a los sistemas DRM por tener el potencial de bloquear el acceso a obras, con frecuencia las críticas se refieren solo o principalmente a las MTP ${ }^{19}$. Sus propósitos son diferentes, la ISGD tiene como objetivo facilitar la distribución y gestión de obras protegidas por derechos de autor, las MTP apuntan a controlar el acceso, la difusión y el uso de obras digitales. Además, la ISGD y las MTP difieren desde la perspectiva de su historia legislativa. Las primeras legislaciones sobre MTP se remontan a la normatividad nacional y regional de finales de los años ochenta y principios de los noventa, especialmente de Estados Unidos, Europa y Japón ${ }^{20}$. La protección legal de las MTP se estableció por primera vez en las normas nacionales, en cambio, la ISGD se estableció primero en tratados internacionales de la OMPI y luego fue implementada en las legislaciones nacionales y en normas regionales. En la siguiente sección se explicará el origen de la protección legal de la ISGD en los Tratados Internet de la OMPI de 1996 y sus discusiones previas.

\subsection{Origen y propósito de la protección legal a la integridad de la ISGD}

La protección legal de la información de derechos de autor anticipó la importancia de los metadatos para la gestión de la propiedad intelectual en las redes digitales mundiales. Tradicionalmente, las normas de derechos de autor han aparecido de forma posterior, como reacción, a los desarrollos tecnológicos. Como lo señalan Jörg Reinbothe y Silke von Lewinski, las normas sobre la ISGD son un raro ejemplo de una regulación que ha precedido en gran medida a los avances tecnológicos y la implementación de la tecnología en la industria cultural ${ }^{21}$. La protección jurídica a la integridad de estos metadatos fue una iniciativa del gobierno de los Estados Unidos antes del uso masivo de Internet ${ }^{22}$. Los documentos base para esta iniciativa fueron el reporte del grupo de trabajo de la infraestructura de la información de los Estados Unidos y el Libro Verde europeo.

\footnotetext{
${ }^{17}$ Las MTP son tecnologías que controlan el acceso o uso a las obras protegidas por el derecho de autor. FICSOR (2010).

${ }^{18}$ FRASER (1997), p. 780; Boyle erróneamente argumentó que: "the distinction between $\S 1201$, covering copyright protection devices, and § 1202, covering copyright management information, might blur and even disappear". BOYLE (1996), p. 108.

${ }^{19}$ FICSOR (2002), p. 258.

${ }^{20}$ En los Estados Unidos por ejemplo con relación a la comunicación por cable: Public Telecommunications Act 47 USC, s 605(e)(4); Audio Home Recording Act (AHRA) 17 USC, s 1002(c). En el Reino Unido, con relación a dispositivos diseñados para eludir sistemas de protección de copia ver: Copyright, Designs and Patents Act 1988, s 296 (UK); en Europe see Directive 91/250/EEC on the Legal Protection of Computer Programs [1991] OJ L122, art. 7(1)(c).

${ }^{21}$ REINBOTHE Y LEWINSKI (2015), p. 179.

${ }^{22}$ FICSOR (2002), p. 12.
} 


\subsubsection{Grupo de trabajo de la infraestructura de la información - Estados Unidos}

En 1993, la administración de Estados Unidos del presidente Bill Clinton, dentro de la Agenda de Acción de su gobierno, creó el grupo de trabajo de la infraestructura de la información (IITF). El IITF ayudó a diseñar las políticas y regulaciones para desarrollar la "superautopista de la información" ${ }^{23}$, hoy en día conocida como Internet. Con esta agenda el gobierno llamó a una acción específica con el fin de $^{24}$ :

[...] explorar formas de identificar y compensar a los titulares de los derechos de autor. El IITF explorará la necesidad de estándares para la identificación de titulares de derechos de autor en sistemas de información electrónicos (por ejemplo, encabezados electrónicos, etiquetas o técnicas de firma). El Grupo de Trabajo también evaluará la necesidad de desarrollar un sistema eficiente para la identificación, licencia y uso de las obras, y para el pago de regalías de productos protegidos por los derechos de autor suministrados o puestos a disposición a través de sistemas electrónicos de información.

Este objetivo delineó el razonamiento detrás de la protección a la integridad de los metadatos de derechos de autor. En el entorno digital, la economía de productos y servicios basados en los derechos de autor es parte de mercados globales. Desde esta perspectiva, el grupo de trabajo abordó la ISGD en su importancia para facilitar la concesión de licencias ${ }^{25}$ y su relación con los sistemas de gestión de derechos de autor (CMS) o gestión de derechos digitales (DRM). En ese contexto, la propiedad intelectual fue un elemento principal para la agenda del gobierno norteamericano. Los dos objetivos relacionados con la propiedad intelectual fueron: evaluar la legislación de derecho de autor para el entorno digital y explorar formas de identificar y compensar a los titulares de los derechos de autor ${ }^{26}$. Se creó entonces un equipo presidido por Bruce Lehman ${ }^{27}$ para proponer enmiendas a la legislación de propiedad intelectual.

En julio de 1994 el grupo de trabajo sobre derechos de propiedad intelectual propuso por primera vez que los metadatos de derechos de autor deberían estar legalmente protegidos. El grupo definió la información de gestión de derechos de autor como la "información asociada con una obra protegida por derechos de autor, que incluye, entre otros, el nombre y otra información de identificación del titular del derecho de autor, los términos y condiciones para los usos de la obra y códigos de identificación, como los números ISBN"28.

En 1995, el IITF publicó su Libro Blanco, el cual incluyó el informe final del grupo de trabajo sobre derechos de propiedad intelectual. El Libro Blanco propuso enmendar la ley de copyright para incluir la protección de la información de gestión de derechos de autor ${ }^{29}$. La redacción de la propuesta legislativa del Libro Verde y el Libro Blanco tuvieron el mismo fundamento y características similares, la diferencia es que la ISGD incluía cualquier otra información que la oficina de derechos de autor definiera por regulación.

\footnotetext{
${ }^{23}$ El artista coreano Nam June Paik acuñó en la década de 1970 el término superautopista de la información, ver PAIK (1995).

24 IITF (1993).

${ }^{25}$ LEHMAN (1995), p. 53.

${ }^{26}$ LEHMAN (1995), p. 11.

${ }^{27}$ Bruce Lehman era en ese momento el "Assistant Secretary of Commerce and Commissioner of Patents and Trademarks". Para más información sobre Bruce Lehman y su rol en la creación y promoción del Libro Blanco ver SAMUELSON (1996).

${ }^{28}$ LEHMAN (1994).

${ }^{29}$ LEHMAN (1995), Appendix 2.
} 
El gobierno de los Estados Unidos justificó la protección de la ISGD al concluir que los metadatos reducirían los costos de transacción de las licencias. Además, estos metadatos ayudarían a crear sistemas de gestión de derechos de autor más eficientes en el entorno digital. El Libro Blanco declaró que "la información de gestión de derechos de autor en la autopista de la información servirá como una especie de 'placa vehicular' para las obras" 30 . Una de las recomendaciones del grupo de trabajo es que los titulares deben usar la IGSD para identificar y localizar fácilmente las obras legítimas protegidas por los derechos de autor ${ }^{31}$.

La creación de nuevos mercados para los productos culturales fue uno de los objetivos de la infraestructura nacional de información. La protección legal de la ISGD tuvo como objetivo proteger el interés de los titulares de derechos de autor y del público. La protección de los intereses públicos consiste en prohibir la circulación y difusión de ISGD falsa; para los consumidores de obras digitales es importante ya que esta información garantiza una forma más cómoda de encontrar obras digitales y usarlas legalmente. La protección a los titulares de derechos de autor consiste en prohibir la eliminación y alteración de la ISGD. Para proteger los intereses de los titulares de los derechos de autor y los consumidores, la ISGD debe ser precisa y confiable. La precisión e integridad de los metadatos de derechos de autor en infraestructuras de información de derechos, como los registros de derechos de autor, es tan importante como la ISGD adjunta a las obras.

El grupo de trabajo de la infraestructura de la información, la Comisión Europea y los delegados que asistieron a la Conferencia de la OMPI justificaron la protección legal a la ISGD y a las MTP dentro de dos áreas principales. En primer lugar, se enfatizó la necesidad de dar soluciones para mitigar los riesgos que los titulares de propiedad intelectual podrían encontrar con el desarrollo de las tecnologías digitales y, en segundo lugar, la superautopista de la información traería nuevas oportunidades de mercado para las industrias culturales. De acuerdo con estos argumentos, la legislación debía proporcionar protección a las tecnologías que podrían minimizar tales riesgos y estimular las tecnologías e infraestructuras para la difusión y explotación de obras digitales. El IITF previó que las MTP y la ISGD podrían crear mecanismos para reducir la piratería y reducir los costos de las licencias. Además, los usos de estas tecnologías proporcionarían nuevos servicios para la distribución digital de obras ${ }^{32}$.

En 1995, por primera vez, se presentó en el Congreso de los Estados Unidos un proyecto de ley que incluyó normas para la protección de la ISGD"33. El proyecto de ley titulado "Protección de derechos de autor en la infraestructura nacional de la información" (NIICPA) siguió las recomendaciones del Libro Blanco. Este proyecto de ley fue altamente controvertido, por ejemplo, 106 profesores de derecho -incluidos prestigiosos académicos de la propiedad intelectual, como Jessica Litman, Pamela Samuelson y Margaret Chon - firmaron una carta abierta oponiéndose al proyecto de ley ${ }^{34}$. Los diferentes sectores que se opusieron al proyecto de ley rechazaron enérgicamente la protección de las medidas tecnológicas de protección ${ }^{35}$ y se criticó a la ISGD por la preocupación de que al ser parte de los sistemas DRM se podría interferir con la privacidad de los usuarios de las obras digitales ${ }^{36}$. Finalmente, el proyecto no fue aprobado. Ante el fracaso en esta

\footnotetext{
${ }^{30}$ LEHMAN (1995), p. 235.

${ }^{31}$ LEHMAN (1995), p. 236.

32 LEHMAN (1995).

${ }^{33}$ NII Copyright Protection Act of 1995, 1995-1996 (bill text 104th Congress, H.R.2441.IH).

${ }^{34}$ BOYLE (1996), p. 60.

${ }^{35}$ Sobre el debate político en contra de las medidas tecnológicas de protección ver el capítulo 3 de HERMAN (2013).

${ }^{36}$ COHEN (1996). En todo caso, la ISGD por sí sola no permite rastrear los comportamientos de los usuarios. Similares y equivocadas preocupaciones sobre el uso de la ISGD y la privacidad fueron repetidos décadas más tarde en otras latitudes, por ejemplo, en Nueva Zelanda. Ver: NZ Vice Chancellors' Committee Standing Committee on Copyright (2007); Internet NZ (2007).
} 
iniciativa legislativa la estrategia del gobierno norteamericano se movió de lo nacional a lo multilateral para introducir este tema en las discusiones de la OMPI, como veremos más adelante.

\subsubsection{Libro Verde - Europa}

En diciembre de 1993, el Consejo Europeo solicitó un estudio destinado a adoptar políticas para la sociedad de la información. Como resultado, en 1994, el Informe Bangemann reconoció que en el entorno digital es una prioridad proteger la propiedad intelectual para el adecuado desarrollo económico. Además, el informe subrayó que la flexibilidad y la eficiencia para obtener licencias de explotación de obras sería un requisito para el crecimiento de una industria multimedia europea ${ }^{37}$. En los años siguientes, los sistemas automáticos de concesión de licencias y la gestión de derechos de autor fueron puntos claves dentro de las discusiones sobre la eficiencia del mercado de explotación digital de obras.

En 1995, la Comisión Europea preparó un Libro Verde sobre el derecho de autor y los derechos conexos en la sociedad de la información ${ }^{38}$. Este documento propuso reformas legales al derecho de autor en relación con las nuevas tecnologías digitales y el uso creciente de la superautopista de la información o la Internet. Informes nacionales precedieron al Libro Verde, principalmente el "Informe Sirinelli" en 1994, que fue un estudio dirigido por Pierre Sirinelli bajo los auspicios del Ministerio de Cultura francés ${ }^{39}$.

El Libro Verde se basó en la convicción de que para el desarrollo de la sociedad de la información en Europa era necesaria una reforma de la propiedad intelectual enfocada en una fuerte protección a los titulares de los derechos ${ }^{40}$. De esta forma, la Comisión Europea se orientó hacia el reconocimiento de altos estándares de protección del derecho de autor. Sin embargo, hubo una preocupación explícita de mantener un equilibrio con los intereses de los usuarios en el ámbito digital ${ }^{41}$. El Libro Verde se centró en los aspectos económicos de la sociedad de la información y la propiedad intelectual, pero, en contraste con el Libro Blanco de los Estados Unidos, el Libro Verde también menciona otras facetas. El Libro Verde abordó la protección del derecho de autor en tres dimensiones: cultural, económica y social. La dimensión cultural hizo hincapié en la importancia del patrimonio cultural común de Europa. Propuso un equilibrio entre la protección del patrimonio cultural y las normas de propiedad intelectual ${ }^{42}$. El énfasis en la protección del patrimonio estaba firmemente arraigado en la idea de que dentro de la sociedad de la información el uso del patrimonio puede conducir a la creación de contenido creativo, nuevos servicios, empleos y beneficios económicos para la comunidad europea en general. Esto presenta una perspectiva basada en la idea del patrimonio cultural como industria.

El Libro Verde se refirió a los metadatos de derechos de autor solo de manera tangencial. Las partes que participaron en la consulta fueron enfáticas sobre el interés en la identificación y gestión de obras en el entorno digital, además de acentuar su posición en contra de las licencias obligatorias y presentar serios cuestionamientos sobre la gestión individual de derechos ${ }^{43}$. El tema de los metadatos se relacionó con dos puntos, en primer lugar, la gestión del derecho de autor y los mecanismos técnicos para la protección y, en segundo lugar, la identificación de los contenidos

\footnotetext{
${ }^{37}$ BANGEMANN GROUP (1994), p. 22.

${ }^{38}$ EUROPEAN COMMISSION (1995).

${ }^{39}$ SIRINELLI (1994).

${ }^{40}$ EUROPEAN COMMISSION (1995), p. 6.

${ }^{41}$ EUROPEAN COMMISSION (1996), p. 2.

${ }^{42}$ EUROPEAN COMMISSION (1995), p. 11.

${ }^{43}$ EUROPEAN COMMISSION (1995), pp. 16-17.
} 
digitales. Ambos puntos fueron discutidos dentro del contexto de la observancia de los derechos de propiedad intelectual.

Ante las dificultades de la gestión de derechos en las creaciones digitales que combinan múltiples obras protegidas por el derecho de autor, por ejemplo, obras multimedia que requieren el uso de obras preexistentes, se requieren tecnologías que permitan a los titulares la identificación y el seguimiento de los diferentes usos que se hacen de sus obras ${ }^{44}$. Sin embargo, el Libro Verde no propuso la protección legal de los metadatos de derechos de autor. Los metadatos de derechos de autor solo estaban analizados como parte de las tecnologías de gestión de derechos que pueden permitir a los titulares identificar, monitorear y controlar el uso de obras digitales ${ }^{45}$. En ese momento se pronosticó que estos usos generarían una gestión más efectiva de derechos, lo cual hoy en día, dos décadas después, se ha empezado a constatar.

La Comisión Europea discutió los sistemas de protección técnica y la identificación de obras en el entorno digital abordando los identificadores digitales (los cuales son metadatos) y los sistemas de gestión de derechos digitales como una nueva oportunidad para los titulares de propiedad intelectual, por ejemplo, para la automatización de la gestión de los derechos ${ }^{46}$. Así mismo, la Comisión percibió la necesidad de crear estándares internacionales para la identificación de obras en el entorno digital, destacando iniciativas de la industria musical y el papel de la Organización Internacional de Estándares (ISO) en la creación de estas normas ${ }^{47}$.

\subsection{Los tratados Internet de la OMPI}

El Libro Blanco de los Estados Unidos y el Libro Verde europeo publicado en 1995 fueron los principales documentos tomados en cuenta para la preparación de los tratados de la OMPI de 1996. Los Tratados Internet de la OMPI tienen su origen inmediato después de la formación de la Organización Mundial del Comercio (OMC) y la adopción del Acuerdo sobre los aspectos de los derechos de propiedad intelectual relacionados con el comercio (ADPIC) en 1994 ${ }^{48}$. La OMPI comenzó a organizar los Tratados Internet una vez que se adoptó el acuerdo sobre los ADPIC ya que coincidió con la aparición de Internet y el uso generalizado de tecnologías digitales, temas que no fueron discutidos dentro de la creación de la $\mathrm{OMC}^{49}$.

Anterior a los Tratados Internet de la OMPI, la única referencia explícita a la protección jurídica de la ISGD figuraba en el Libro Blanco de los Estados Unidos y en el reporte del gobierno japonés relacionado con el derecho de autor y obras multimedia ${ }^{50}$. Los artículos sobre la protección jurídica de la ISGD en los tratados de la OMPI fueron propuestos por el gobierno de los Estados Unidos $^{51}$. Las propuestas posteriores y los comentarios de Canadá, América Latina, la Comunidad Europea, Japón y China no proporcionaron ningún elemento nuevo a la discusión. Sin embargo, la propuesta de Corea del Sur presentó elementos técnicos al debate que desafortunadamente no fueron atendidos.

\footnotetext{
${ }^{44}$ EUROPEAN COMMISSION (1995), p. 28.

${ }^{45}$ EUROPEAN COMMISSION (1995), p. 75.

${ }^{46}$ EUROPEAN COMMISSION (1995), p. 37.

${ }^{47}$ EUROPEAN COMMISSION (1995), p. 82.

${ }^{48}$ ACUERDO SOBRE LOS ASPECTOS DE LOS DERECHOS DE PROPIEDAD INTELECTUAL RELACIONADOS CON EL COMERCIO (1994).

${ }^{49}$ FICSOR (2002), p. 25.

50 WORKING GROUP OF THE SUBCOMMITTEE ON MULTIMEDIA COPYRIGHT COUNCIL (1995), p. 42.

${ }^{51}$ FICSOR (2002), p. 391.
} 
Corea del Sur propuso que una autoridad nacional o un organismo internacional debería estandarizar la información de gestión de derechos ${ }^{52}$. Este enfoque proporciona claridad para identificar la ISGD y delimitar los metadatos legalmente protegidos. La propuesta de los Estados Unidos y el texto final aprobado por la OMPI definen la ISGD, pero no suministran ningún elemento técnico con el cual se pueda identificar claramente la ISGD. En otras palabras, la fórmula adoptada por la OMPI proporciona una definición vaga e imprecisa de la ISGD. El texto de los tratados de la OMPI establece que la integridad de los metadatos que proporcionan información de derechos de autor debe estar protegida, pero no define quién debe crear los metadatos, ni cómo distinguir la ISGD legalmente protegida de otros metadatos que proporcionan información relacionada con el derecho de autor ${ }^{53}$. El enfoque que propuso Corea del Sur es conveniente desde una perspectiva técnica y jurídica, ya que proporciona claridad para definir qué metadatos están dentro del alcance de la ley.

A finales de 1996, la sesión plenaria de la Conferencia Diplomática de la OMPI aprobó los textos presentados por la delegación de los Estados Unidos respecto a la ISGD ${ }^{54}$. Los tratados de la OMPI establecieron que las partes contratantes deberían proporcionar protección legal para garantizar la integridad de la ISGD. Estas disposiciones tienen como objetivo castigar dos eventos diferentes: primero, la remoción o alteración no autorizada de la ISGD, y segundo, la distribución y comunicación pública de obras donde la ISGD ha sido removida o alterada sin autorización ${ }^{55}$.

\subsection{Implementación nacional}

Después de los Tratados Internet de la OMPI, la protección a la información sobre la gestión de derechos comenzó a ser implementada en la legislación nacional. Como se indicó en los párrafos anteriores, antes de estos tratados no había ninguna legislación sobre la protección legal a la integridad de la ISGD ${ }^{56}$. El proceso de difusión legal en materia de la protección a la ISGD se ha llevado a cabo por la implementación de los Tratados Internet de la OMPI, por medio de tratados de libre comercio (TLC) que contienen disposiciones relativas a las ISGD o por la actualización de la legislación nacional de derechos de autor al entorno digital. Por ejemplo, Colombia ratificó los tratados de la OMPI en 2000 y ese mismo año estableció la protección de la ISGD en su código penal ${ }^{57}$. En el 2018, Colombia implementó algunas normas derivadas de sus obligaciones adquiridas por el TLC con los Estados Unidos, en esta reforma se incorporó una definición de la ISGD y se añadió a su protección legal la responsabilidad civil como una vía diferente a la penal ${ }^{58}$. Por otro lado, Nueva Zelanda no ha firmado los tratados de Internet ni tiene obligaciones internacionales relacionadas con la ISGD, pero en 2008 y 2011 actualizó su legislación de derecho de autor para hacer frente a los desafíos digitales, incluidas algunas normas para la protección legal a la ISGD ${ }^{59}$.

Estados Unidos fue el primer país en implementar la protección a la ISGD con la Digital Millennium Copyright Act (DMCA) de 1998. La ISGD en la legislación de los Estados Unidos se denomina información de gestión de derechos de autor (Copyright Management Information), la

\footnotetext{
52 DELEGATION OF THE REPUBLIC OF KOREA (1996).

53 Por ejemplo, los bibliotecarios producen metadatos de derechos de autor durante la gestión de colecciones; según los tratados de la OMPI estos metadatos encajan dentro de la definición de ISGD.

${ }^{54}$ FICSOR (2002), p. 406.

55 TODA, art. 12; WPPT, art. 19.

${ }^{56}$ REINBOTHE Y LEWINSKI (2015), p. 179.

${ }^{57}$ Código Penal, art 272 (Colombia).

58 Ley 1915 de 2018, art. 12.

${ }^{59}$ Copyright (New Technologies) Amendment Act 2008; Copyright (Infringing File Sharing) Amendment Act 2011.
} 
cual es definida de la siguiente manera: "información transmitida en relación con copias o fonogramas de una obra o representaciones o exhibiciones de una obra"60. Dicha información identifica el autor, la obra, el titular de copyright, el artista, escritor, actor, director de las obras audiovisuales, la información de los avisos de derechos de autor, los términos y condiciones para el uso de la obra, la identificación de números o símbolos que hacen referencia a dicha información o enlaces a dicha información y otra información señalada por la oficina de derechos de autor ${ }^{61}$. Esta definición implementó las obligaciones derivadas de ambos tratados de Internet de la OMPI y va más allá debido a que incluye la información para identificar a los artistas intérpretes o ejecutantes y directores de obras audiovisuales.

Después de los Estados Unidos, múltiples países han adoptado la protección a la integridad de la ISGD. Las diferencias en la legislación nacional son mínimas y no son significativas para efectos de este estudio. Los académicos dedicados al derecho de autor han prestado poca atención a la $I_{S G D}{ }^{62}$ y la jurisprudencia sobre este tema ha sido escasa ${ }^{63}$. Como hemos visto, el desarrollo de redes de información digital motivó esta nueva clase de derechos y de manera concomitante las industrias de derechos de autor, las comunidades académicas y los profesionales de ciencias de la información han estado trabajando en la estandarización de los metadatos de derechos de autor y en crear soluciones para la gestión de la propiedad intelectual; lo cual analizaremos en la siguiente sección.

\section{Infraestructuras de metadatos de derechos de autor}

En la era digital, las bases de datos se están convirtiendo en parte fundamental de las políticas públicas y legislación para la gestión de derechos de autor. Así, por ejemplo, en el 2018, en la más reciente reforma de derechos de autor de los Estados Unidos, por mandato legal se debe crear una base de datos para la adecuada identificación de titulares y distribución de regalías en la explotación de música en streaming y otros ambientes digitales ${ }^{64}$. Como lo ha señalado Molly Shaffer Van Houweling, las soluciones tecnológicas para resolver los retos que enfrenta el derecho de autor deben ser parte fundamental de una reforma a las políticas de la propiedad intelectual ${ }^{65}$. En este sentido, las infraestructuras de información de derechos, las normas de catalogación y los diccionarios de derechos ${ }^{66}$ apuntan a agregar y estandarizar la información sobre derechos provenientes de diferentes fuentes, tales como contratos, acuerdos de adquisición, bases de datos, usuarios, académicos, organizaciones de gestión colectiva y otras partes interesadas.

Las herramientas tecnológicas anteriormente mencionadas tienen como objetivo crear y gestionar metadatos autoritativos de derechos que proporcionen información precisa para determinar el estatus de derechos de autor de las obras en repositorios digitales (es decir,

\footnotetext{
${ }^{60}$ Copyright Law of the United States of America 17 USC, s 1202 (C).

${ }^{61}$ Section $1202(C)$.

${ }^{62}$ Mark Perry compara las normas relacionadas con la ISGD de Nueva Zelanda, Japón, la Unión Europea y los Estados Unidos, ver: PERRY (2010) 304.

${ }_{63}$ Para una mirada general de la jurisprudencia en los Estados Unidos relacionada con la ISGD ver: LIM (2011).

${ }^{64}$ Orrin G. Hatch-Bob Goodlatte Music Modernization Act, 2018.

${ }^{65}$ SHAFFER VAN HOUWELING (2012), p. 1501.

${ }^{66}$ Los diccionarios de metadatos en el área de la organización de la información y el conocimiento se refieren al uso de términos, temas y definiciones siguiendo reglas acordadas para describir un recurso de información. Un diccionario de derechos se centra en términos, temas y definiciones relacionadas con los derechos y el estatus legal de los recursos de información. El objetivo principal de un diccionario de metadatos de derechos es establecer claridad para investigar, registrar, recuperar e interpretar información legal importante para la gestión de un recurso digital. WHALEN (2009).
} 
determinar si la obra se encuentra protegida por el derecho de autor o en el dominio público), sus titulares y otra información legal relevante. Estos proyectos están motivados por una racionalidad económica que busca una mayor eficiencia en la gestión de derechos y regalías ${ }^{67}$. Estos metadatos permiten tomar decisiones informadas y evaluar los riesgos legales al copiar y distribuir copias de obras intelectuales ${ }^{68}$. Por ejemplo, la información de derechos sirve para identificar obras en el dominio público y así decidir qué colecciones podrían digitalizarse sin riesgos legales o con riesgos legales mínimos.

\subsection{Derechos de autor y estándares de catalogación}

MARC (Machine-Readable Cataloging) es el estándar prevalente para la catalogación en las bibliotecas en todo el mundo. Inicialmente MARC carecía de una adecuada estructura para registrar información de derechos de autor. Esto creó un problema para las bibliotecas que deseaban implementar estrategias de registro de derechos en sus catálogos y sistemas de metadatos. MARC carecía de un campo específico para registrar información sobre el historial de derechos de autor, el estado de derecho de autor o la interpretación de derechos de autor. Los campos de MARC relacionados con derechos de autor, como el número de derechos de autor o depósito legal (campo 017), las restricciones de acceso (campo 506) o los términos de uso y reproducción (campo 540) no proporcionaban suficiente información legal sobre los recursos.

En el año 2008, la Biblioteca del Congreso agregó a la estructura de MARC el campo 542 titulado "Información relacionada con el estado de los derechos de autor" (tabla 1). Este campo está destinado a crear metadatos que suministran información de derechos de autor sobre los recursos que poseen y gestionan las bibliotecas. La eficiencia en la toma de decisiones es el fundamento principal para crear este nuevo campo en MARC, así las razones para crear metadatos de derechos de autor son económicas, legales y de gestión. Las razones económicas están relacionadas con la eficiencia en la recuperación de información, las legales para evaluar riesgos jurídicos y las de gestión para la toma de decisiones frente a la accesibilidad y digitalización.

Tabla 1. Elementos de datos en el Campo 542 de MARC: "Información relacionada con el estado de derechos de autor"

\begin{tabular}{|l|l|}
\hline \multicolumn{1}{|c|}{ Creador } & \multicolumn{1}{c|}{ Titular de derechos de autor } \\
\hline Fecha de defunción del creador & $\begin{array}{l}\text { Información de contacto del titular de } \\
\text { derechos de autor }\end{array}$ \\
\hline Creador corporativo & Declaración de copyright \\
\hline Fecha de creación & Fecha de renovación de copyright \\
\hline Fecha de copyright & Fecha de publicación \\
\hline Estado de copyright & Estado de publicación \\
\hline Fecha de la investigación & País de publicación o creación \\
\hline $\begin{array}{l}\text { Agencia que provee la } \\
\text { información }\end{array}$ & $\begin{array}{l}\text { Jurisdicción de la evaluación de derechos } \\
\text { de autor }\end{array}$ \\
\hline Fuente de información & Identificador uniforme de recursos \\
\hline Editor & Enlace \\
\hline
\end{tabular}

\footnotetext{
${ }^{67}$ WHALEN (2009), p. 20.
}

${ }^{68}$ WHALEN (2009), p. 18. 


\begin{tabular}{|l|l|}
\hline Nota & Enlace de campo y número de secuencia \\
\hline Materiales especificados & \\
\hline
\end{tabular}

\subsection{Información para la recuperación de recursos}

Los metadatos de derechos de autor sirven para recuperar información. Los nuevos servicios comerciales permiten buscar y recuperar información de acuerdo con el estatus de derecho de autor de las obras o licenciamientos específicos. Estos servicios integran metadatos de derechos de autor en sus campos de búsqueda. Por ejemplo, en el proyecto de Flickr, titulado "The Commons", es posible buscar y recuperar fotos con metadatos que indican licencias específicas, tales como Creative Commons (CC) ${ }^{69}$. Múltiples instituciones de memoria de todo el mundo contribuyen a este proyecto y cada institución de memoria establece su propia información de derechos para sus colecciones.

Existe una tendencia emergente y creciente de utilizar los metadatos de derechos de autor como un campo en la recuperación y búsqueda de información, especialmente para colecciones de patrimonio digital. Por ejemplo, el Ministerio de Educación de Brasil creó el proyecto Dominio Público ${ }^{70}$, el cual proporciona acceso al material identificado en el dominio público a través de metadatos de derechos que indican esa condición. La biblioteca digital europea, Europeana, permite refinar la búsqueda de material de patrimonio digital teniendo como criterio de búsqueda el estatus de derecho de autor, por ejemplo, se recupera un material identificando si se encuentra en dominio público, si es de acceso pago, acceso restringido, si tiene derechos reservados o si sus derechos de autor son desconocidos ${ }^{71}$.

\subsection{Infraestructuras de información de derechos}

Las bases de datos de derechos no son nuevas en el derecho de autor. Los registros de derechos y los catálogos de derechos de autor han existido desde el siglo XIX. En 1825, William y Seth Elliot de la Oficina de Patentes de los Estados Unidos publicaron la primera lista de libros protegidos por el derecho de autor en dicho país ${ }^{72}$. Desde el siglo XX las organizaciones de gestión colectiva han sido muy activas en la creación de bases de datos de derechos de autor para el recaudo y distribución de regalías a nivel local, regional e internacional ${ }^{73}$.

Las infraestructuras de información de derechos (Rights Information Infrastructures, RII) es un concepto que surgió en el siglo XXI. Las RII son ${ }^{74}$ :

Sistemas basados en estándares, que están concebidos para ser utilizados en cualquier contexto, con el fin de gestionar la información de derechos. El valor principal es proporcionar interoperabilidad entre los recursos existentes y fomentar la recopilación de datos adicionales o el enriquecimiento de los datos existentes dentro de una red.

Las infraestructuras de información de derechos se diseñan desde un enfoque de gestión de la información. La interoperabilidad y el intercambio de recursos a través de las instituciones son

\footnotetext{
${ }^{69}<$ www.flickr.com/commons/usage/>

${ }^{70}<$ www.dominiopublico.gov.br/>

${ }^{71}<$ www.europeana.eu/portal/usingeuropeana_search.html>

72 DUNNE Y ROGERS (1960), p. 55.

${ }_{73}^{73}$ Por ejemplo, las bases de datos de la confederación internacional de autores y compositores (CISAC).

${ }^{74}$ ATTANASIO (2010), p. 248.
} 
las principales características de las RII, estas son las diferencias conceptuales claves entre las bases de datos de derechos tradicionales y las infraestructuras de información de derechos.

De manera concomitante a las instituciones de la memoria, organizaciones privadas y públicas están creando infraestructuras de información de derechos con el objetivo de mejorar la gestión de los derechos de autor a través de plataformas digitales. Gobiernos nacionales están tomando iniciativas para crear estas infraestructuras como parte de sus planes para promover la economía digital. Por ejemplo, el "Copyright Hub" en el Reino Unido es la plataforma digital de intercambio de información de derechos de autor ${ }^{75}$. Esta plataforma fue creada en el 2013 con apoyo y fondos del gobierno. Su principal objetivo es hacer del licenciamiento de derechos de autor algo fácil y simple. Por otro lado, la industria tecnológica enfocada en la economía digital ha creado sus propias bases de datos de derechos de autor, como es el caso del registro de libros dentro del proyecto Google Books ${ }^{76}$.

En el plano internacional, la OMPI junto con la industria de la música trabaja en el Registro Internacional de Música (IMR) y en la base de datos de repertorios mundiales ${ }^{77}$, para mejorar la identificación de derechos de autor, la obtención de licencias y el funcionamiento de las organizaciones de gestión colectiva ${ }^{78}$. Estos registros proporcionan eficiencias para la búsqueda de información de derechos de autor, la identificación de los titulares de derechos de autor y suministrar información para contactar a los titulares de una forma fácil y centralizada. Un desafío de estas infraestructuras es la necesidad de mantener información actualizada. Los derechos podrían cambiar con el tiempo, por ejemplo, debido al vencimiento de la licencia, los cambios en los términos de la licencia y el vencimiento de los derechos de autor. Además, la información sobre los titulares de los derechos de autor podría cambiar, como la información de contacto, la transferencia de la titularidad y la existencia de diferentes titulares sobre derechos diferentes que recaen en una misma obra.

\subsubsection{Obras huérfanas e infraestructuras de información de derechos}

Las infraestructuras de información de derechos en instituciones públicas son principalmente una respuesta al problema de la digitalización de obras huérfanas. Las obras huérfanas son aquellas en las que no se puede identificar o localizar al titular de derechos de autor, lo cual resulta por la inadecuada gestión de derechos y por la falta de información sobre los titulares. Asociaciones de autores y editores, bibliotecas nacionales, organizaciones de derechos de reproducción, instituciones culturales, industrias culturales y desarrolladores de tecnología están trabajando de manera conjunta en la creación de infraestructuras para la solución de los problemas relacionados con las obras huérfanas.

Las instituciones nacionales de memoria están creando infraestructuras de información de derechos como bases de datos y registros de derechos de autor para gestionar sus colecciones digitales. En la Unión Europea, el Registro Accesible de Información de Derechos y Obras Huérfanas (ARROW, Accessible Registries of Rights Information and Orphan Works towards Europeana) ${ }^{79}$ es un proyecto pionero en el intercambio colaborativo de metadatos de derechos de autor. ARROW es

\footnotetext{
${ }^{75}$ http://www.copyrighthub.org

${ }^{76}$ BAND (2009).

77 GARNETT (2012).

${ }^{78}$ GERVAIS (2010).

${ }^{79}$ FORWARD (Framework for a EU-Wide Audiovisual Orphan Works Registry) es un proyecto que comenzó en 2013 para crear un sistema estandarizado y de alcance internacional para evaluar y registrar el estado de los derechos de las obras audiovisuales con especial énfasis en obras huérfanas. <http://project-forward.eu>
} 
una infraestructura de administración de información de derechos enfocada en libros publicados. ARROW proyecta resolver en los próximos años "el agujero negro del siglo XX" en las colecciones digitales europeas. ARROW es el centro de intercambio de información sobre derechos de Europeana con el objetivo de permitir el acceso y la digitalización de libros. Además, por mandato legal, se creó a nivel regional la base de datos europea sobre obras huérfanas ${ }^{80}$.

En estos contextos, las estrategias de metadatos de derechos de autor apuntan a crear e intercambiar información de derechos dentro de cada institución y compartir la información entre las organizaciones. Por ejemplo, las bibliotecas nacionales en Europa han negociado acuerdos con los titulares de derechos de autor para tener acceso a fuentes de metadatos ${ }^{81}$. Esto nos muestra con claridad que la protección legal a la integridad de la ISGD por sí sola no es suficiente para crear ambientes digitales que faciliten el licenciamiento, la identificación de obras y resuelvan el problema de las obras huérfanas. El ambiente digital exige que las instituciones de memoria se involucren comprometidamente con la gestión de la propiedad intelectual desde el punto de visto tecnológico como legal.

\section{Conclusión}

La protección legal a la integridad de la información de gestión de derechos es parte de la gobernanza global de Internet y fue diseñada específicamente como parte de la regulación de la información en las redes digitales. Desde la década de los noventa del siglo XX, los metadatos de derechos de autor fueron identificados por el gobierno de los Estados Unidos como un elemento importante para la gestión de la propiedad intelectual y la explotación de las obras en el mercado digital, logrando que la OMPI en 1996 adoptara su protección con los tratados Internet. Más adelante, estas medidas han empezado a ser implementadas alrededor de todo el mundo. Sin embargo, la definición legal de la ISGD es imprecisa, lo cual dificulta delimitar con precisión su alcance.

El objetivo principal de la ISGD es facilitar el licenciamiento, la recolección y la distribución de regalías en la explotación de obras protegidas por derechos de autor. La ISGD constituye una nueva capa de protección legal para los titulares de derechos de autor. Esta capa busca estimular las eficiencias del mercado a través de una identificación adecuada de los contenidos digitales. Además, al facilitar el acceso a la información sobre los titulares de derechos, la ISGD es una de las principales herramientas para evitar que continúen surgiendo obras huérfanas.

Por otro lado, la protección a la ISGD debe pensarse como una medida para promover la creación de infraestructuras de metadatos de derechos de autor y sistemas digitales de gestión de derechos que operan con metadatos de propiedad intelectual adjuntos o conexos a los recursos digitales. La interoperabilidad de los metadatos de derechos de autor permite maximizar el acceso en línea, la gestión de regalías, la gestión de derechos digitales, la concesión de licencias, la autorización de derecho, la recuperación de información, facilitar que las obras en el dominio público sean más accesibles y cualquier proceso de digitalización más eficiente. Como reflexión final, en el contexto de nuestra región, debemos advertir que es importante que los países latinoamericanos preocupados en estimular sus economías digitales incluyan dentro de sus políticas de propiedad intelectual la promoción de infraestructuras de información sobre la gestión de derechos de autor de la mano con la protección efectiva a los metadatos legales.

\footnotetext{
${ }^{80}$ Directive 2012/28/EU on Certain Permitted Uses of Orphan Works [2012] OJ L299, art. 3(6).

81 LAURSEN (2012), p. 154.
} 


\section{BIBLIOGRAFÍA CITADA}

ANDERS, DANNY; RAIS, RAZI; COBBAN, CÉDRIC Y MOPSIK, EUGENE (2018): Show me the Money: Bringing Transparency to Residuals \& Royalty Payments (Copyright Clearance Center).

ATTANASIO, PIERO (2010): "Rights Information Infrastructures and Voluntary Stakeholders Agreements in Digital Library Programmes", en: Italian Journal of Library and Information Science (Vol. 1), pp. 237-248.

BAND, JONATHAN (2009): "The Long and Winding Road to the Google Books Settlement", en: J. Marshall Rev. Intell. Prop. L. (Vol. 9), pp. 227-329.

BANGEMANN GROUP (1994): "Report on Europe and the Global Information Society", en: Bulletin of the European Union (Supplement 2, $\mathrm{N}^{\circ}$ 94).

BENNETT, COLIN; PARSONS, CHRISTOPHER Y MOLNAR, ADAM (2014): "Forgetting, Non Forgetting and QuasiForgetting in Social Networking: Canadian Policy and Corporate Practice", en: Gutwirth, Serge; Leenes, Ronald y Hert, Paul, Reloading Data Protection Multidisciplinary Insights and Contemporary Challenges (Nueva Jersey, Springer), pp. 41-59.

BOSSTRAETEN, G Y VANHYFTE, T (1997): “Authenticity of Authorship and Work, Belgique", en: Marcel Dellebeke, ALAI Study Days, Copyright in Cyberspace, Copyright and the Global Information Infrastructure (Ámsterdam, Otto Cramwinckel).

BOYLE, JAMES (1996): “Intellectual Property Policy Online: a Young Person's Guide”, en: Harvard Journal of Law \& Technology (Vol. 10, N¹), pp. 48-111.

CAMERON, CATHERINE (2013): "Reinvigorating U.S. Copyright with Attribution: How Courts Can Help Define the Fair Use Exception to Copyright by Considering the Economic Aspects of Attribution", en: Berkeley Journal of Entertainment and Sports Law (Vol. 2, №1), pp. 130-152.

COHEN, JULIE (1996): "A Right to Read Anonymously: A Closer Look at "Copyright Management" in Cyberspace", en: Connecticut Law Review (Vol. 28), pp. 981-1039.

COYLE, KAREN (2004): Rights Expression Languages (Washington, Library of Congress).

(2005): “Descriptive Metadata for Copyright Status”, en: First Monday (Vol. 10, N¹).

DELEGATION OF THE REPUBLIC OF KOREA (1996): Amendment to Article 14 of Draft Treaty N¹ (Geneva, WIPO, Diplomatic Conference on Certain Copyright and Neighbouring Rights Questions, CRNR/DC/50).

DellebeKe, MARCEL (1997): ALAl Study Days, Copyright in Cyberspace, Copyright and the Global Information Infrastructure (Ámsterdam, Otto Cramwinckel). 
DIETZ, ADOLF (1997): "Authenticity of Authorship and Work, General Report", en: Dellebeke, Marcel, ALAI Study Days, Copyright in Cyberspace, Copyright and the Global Information Infrastructure (Ámsterdam, Otto Cramwinckel).

DUNNE, ELIZABETH Y ROGERS, JOSEPH (1960): The Catalogue of Copyright Entries (Washington, Government Printing Office).

DUSOLLIER, SÉVERINE (2003): "Some Reflections on Copyright Management Information and Moral Rights", en: The Columbia Journal of Law \& the Arts (Vol. 25), pp. 377-399.

EUROPEAN COMMISSION (1996): Follow-Up to the Green Paper on Copyright and Related Rights in the Information Society COM/96/568.

$\mathrm{COM} / 95 / 382$.

(1995): Green Paper on Copyright and Related Rights in the Information Society

FARRELL, PAUL (2014): Australian Government Metadata Requests Far Higher than Disclosed (London, The Guardian).

FICSOR, MIHÁLY (2002): The Law of Copyright and the Internet, The 1996 WIPO Treaties, their Interpretation and Implementation (New York, Oxford University Press).

(2010): “Protection of DRM under the WIPO 'Internet Treaties': Interpretation, Implementation and Application", en: Stamatoudi, Irini, Copyright Enforcement and the Internet (Alphen aan den Rijn, Wolters Kluwer).

FRANCHI, E Y MOYSE, P (1997): "Authenticity of Authorship and Work, Canada", en: Dellebeke, Marcel, ALAI Study Days, Copyright in Cyberspace, Copyright and the Global Information Infrastructure (Ámsterdam, Otto Cramwinckel).

FRASER, STEPHEN (1997): "The Copyright Battle: Emerging International Rules and Roadblocks on the Global Information Infrastructure", en: The John Marshall Journal of Computer and Information Law (Vol. 15), pp.759-818.

GARNETT, NICHOLAS (2012): Study on the Role and Functions of the International Music Registry (Geneva, WIPO).

GERVAIS, DANIEL (2010): "Collective Management of Copyright: Theory and Practice in the Digital Age", en: Gervais, Daniel Collective, Management of Copyright and Related Rights (Alphen aan den Rijn, Wolters Kluwer).

HENDERSON, SANDRA Y WALKER, MATTHEW (2008): Development of a Rights Management System for the National Library of Australia's Collections (Melbourne, VALA Conference).

HERMAN, BILL (2013): The Fight Over Digital Rights: The Politics of Copyright and Technology (New York, Cambridge University Press). 
HJøRLAND, BIRGER (2000): “Documents, Memory Institutions and Information Science”, en: Journal of Documentation (Vol. 56, N¹), pp. 27-41.

IITF (1993): The National Information Infrastructure: Agenda for Action (Washington, NII).

INTERNET NZ (2007): Submission on The Copyright (New Technologies and Performers Rights) (Wellington, Bill).

JUDGE, ELIZABETH Y SCASSA, TERESA (2010): "Intellectual Property and the Licensing of Canadian Government Geospatial Data: an Examination of GeoConnections' Recommendations for Best Practices and Template Licences", en: The Canadian Geographer (Vol. 54, N³), pp. 366-374.

KIM, YUNHYONG, FARELL, TRACIE Y ROSS, SEAMUS (2013): Development of the Digital Rights Management Policy (Thessaloniki, BlogForever Consortium).

KROES, NEELIE (2011): Addressing the Orphan Works Challenge Launch of ARROW (Bruselas, Speech/11/163, IFRRO).

LAURSEN, DITTE; FIEDLER CHRISTIANSEN, KÅRE Y LUNDEGÅRD OLSEN, LARS (2012): "Management of Metadata for Digital Heritage Collections", en: Microform \& Digitization Review (Vol. 41) pp. 151-158.

LEHMAN, BRUCE (1994): Intellectual Property and the National Information Infrastructure A Preliminary Draft of the Report of the Working Group on Intellectual Property Rights (Washington, NII).

(1995): Report of the Working Group on Intellectual Property Rights (Washington, NII).

LESCURE, PIERRE (2013): Contribution aux Politiques Culturelles à l'ère Numérique (París, Ministère de la Culture et de la Communication).

LIM, SUSUK (2011): "A Survey of the DMCA's Copyright Management Information Protections: The DCMA's CMI Landscape After All Headline News and McClatchey", en: Washington Journal of Law, Technology \& Arts (Vol. 6), pp. 297-309.

LIPINSKI, TOMAS (2013): The Librarian's Legal Companion for Licensing Information Resources and Services (Chicago, Neal-Schuman Publishers, ALA).

LITMAN, JESSICA (1996): “Revising Copyright Law for the Information Age”, en: Oregon Law Review (Vol. 75).

NATIONAL LIBRARY OF AUSTRALIA (2003): Guidelines for the Preservation of Digital Heritage (París, UNESCO $\mathrm{Cl}-2003 / \mathrm{WS} / 3)$.

NISO (2004): Understanding Metadata (Bethesda, NISO Press). 
NZ VICE CHANCELLORS' COMMITTEE STANDING COMMITTEE ON COPYRIGHT (2007): Submission on The Copyright (New Technologies and Performers Rights) (Wellington, Bill).

OESCH, R (1997): "Authenticity of Authorship and Work, Finland", en: Dellebeke, Marcel, ALAl Study Days, Copyright in Cyberspace, Copyright and the Global Information Infrastructure (Ámsterdam, Otto Cramwinckel).

OXFORD (2018): Oxford English Dictionary (Oxford, Oxford University Press).

PAIK, NAM JUNE (1995): The Electronic Super Highway: Travels with Nam June Paik (Fort Lauderdale: Carl Solway Gallery).

PALLANTE, M (1997): "Authenticity of Authorship and Work, USA", en: Dellebeke, Marcel, ALAI Study Days, Copyright in Cyberspace, Copyright and the Global Information Infrastructure (Ámsterdam, Otto Cramwinckel).

PERRY, MARK (2010): "The Protection of Rights Management Information: Modernization or Cup Half Full?", en: Geist, Michael, From "Radical Extremism" to "Balanced Copyright": Canadian Copyright and the Digital Agenda (Toronto, Irwin Law).

POLLAUD-DULIAN, F (1997): "Authenticity of Authorship and Work, France", en: Marcel Dellebeke, ALAI Study Days, Copyright in Cyberspace, Copyright and the Global Information Infrastructure (Ámsterdam, Otto Cramwinckel).

QUAEDVLIEG, ANTOON (1997): "Authenticity of Authorship and Work, The Netherlands", en: Dellebeke, Marcel, ALAl Study Days, Copyright in Cyberspace, Copyright and the Global Information Infrastructure (Ámsterdam, Otto Cramwinckel).

REINBOTHE, JÖRG Y VON LEWINSKI, SILKE (2015): The WIPO Treaties on Copyright, a Commentary on the WCT, the WPPT, and the BTAP (Oxford, Oxford University Press).

ROBINTON, LILY (2010): “Courting Chaos: Conflicting Guidance from Courts Highlights the Need for Clearer Rules to Govern the Search and Seizure of Digital Evidence", en: Yale Journal of Law and Technology (Vol. 12), pp. 311-326.

SAMUELSON, PAMELA (1996): The Copyright Grab (San Francisco, Wired).

SCOTT, ELISE (2015): "Senate Passes Controversial Metadata Laws", en: The Sydney Morning Herald (26 de marzo).

SHAFFER VAN HOUWELING, MOLLY (2012): "Atomism and Automation", en: Berkeley Technology Law Journal (Vol. 27, N.ํ 3), pp. 1471-1501.

SIRINELLI, PIERRE (1994): Industries Culturelles et Nouvelles Techniques (París: Ministère de la Culture et de la Francophonie). 
StROWEL, ALAIN (2000): "Links, Metatags: A Copyright and Trademark Law Perspective", en: Hansen, Hugh, International Intellectual Property Law and Policy (New York, Juris Publishing), Vol 4.

SUNDARA RAJAN, MIRA (2011): Moral Rights: Principles, Practice and New Technology (Oxford, Oxford University Press).

TAYLOR, LENORE Y HURST, DANIEL (2015): “Malcolm Turnbull Says Access to Journalists' Metadata 'a Special Case'", en: The Guardian, London (19 marzo de 2015).

WESCOTT, LAWRENCE (2007): "The Increasing Importance of Metadata in Electronic Discovery", en: Richmond Journal of Law \& Technology (Vol. 14, N. ${ }^{\circ}$ ), pp. 1-24.

Whalen, MAUREen (2009): "Developing a Rights Metadata Dictionary for Digital Surrogates", en: Journal of Library Metadata (Vol. 9), pp. 15-35.

WORKING GROUP OF THE SUBCOMMITTEE ON MULTIMEDIA COPYRIGHT COUNCIL (1995): Study of Institutional Issues Regarding Multimedia (Tokyo, Agency for Cultural Affairs).

ZINS, CHAIM (2007): "Conceptual Approaches for Defining Data, Information, and Knowledge", en: Journal of the American Society for Information Science and Technology (Vol. 58, N. ${ }^{\circ} 4$ ), pp. 479493.

\section{NORMAS JURÍDICAS CITADAS}

ORRIN G. HATCH-BOB GOODLATTE MUSIC MODERNIZATION ACt. 2018.

LEY 1915, Por la cual se modifica la Ley 23 de 1982 y se establecen otras disposiciones en materia de derechos de autor y derechos conexos. 12 de julio de 2018 (Colombia).

TELECOMMUNICATIONS (INTERCEPTION AND ACCESS) AMENDMENT (DATA RETENTION). Act 2015 (Australia).

OMPI TRATADO DE BEIJING SOBRE INTERPRETACIONES Y EJECUCIONES AUDIOVISUALES. 2012.

DIRECTIVE 2012/28/EU ON CERTAIN PERMITTED USES OF ORPHAN WORKS. 2012, OJ L299.

COPYRIGHT (INFRINGING FILE SHARING) AMENDMENT Act. 2011 (Nueva Zelanda).

COPYRIGHT (NEW TECHNOLOGIES) AMENDMENT Act. 2008 (Nueva Zelanda).

LEY 599, Código Penal. 24 de julio de 2000 (Colombia).

WIPO COPYRIGHT TREATY. 1996.

WIPO PERFORMANCES AND PHONOGRAMS TREATY. 1996.

LEY FEDERAL DEL DERECHO DE AUTOR. 24 de diciembre de 1996 (México). 
DIRECTIVE 96/9/EC 1996 ON THE LEGAL PROTECTION OF DATABASES. 1996, OJ L 077.

NII COPYRIGHT PROTECTION ACT OF 1995. 1995-1996 (bill text 104th Congress, H.R.2441.IH).

ACUERDO SOBRE LOS ASPECTOS DE LOS DERECHOS DE PROPIEDAD INTELECTUAL RELACIONADOS CON EL COMERCIO. 15 de abril de 1994.

COPYRIGHT ACT. 1994 (Nueva Zelanda).

DIRECTIVE 91/250/EEC ON THE LEGAL PROTECTION OF COMPUTER PROGRAMS. 1991, OJ L122.

COPYRIGHT, DESIGNS AND PATENTS ACT. 1988, s 296 (UK);

CONVENIO DE BERNA PARA LA PROTECCIÓN DE LAS OBRAS LITERARIAS Y ARTÍSTICAS. 9 de septiembre de 1886, última modificación en 1979.

COPYRIGHT LAW OF THE UNITED STATES OF AMERICA. 17 USC.

PUBLIC TELECOMMUNICATIONS Act. 47 USC.

AUDIO HOME RECORDING Act. AHRA, 17 USC. 